\title{
Numerical Study of Flow Past Three Rectangular Rods at Unequal Gap Spacing
}

\author{
S. Ul. Islam' ', R. Manzoor'2, M. Zahid'', S. Kulsoom² and U. Kausar² \\ 'Department of Mathematics, COMSATS University of Information Technology, Islamabad-44000, Pakistan \\ ²Department of Mathematics, SBK Women's University, Quetta, Balochistan, Pakistan; \\ raheela_manzoor@yahoo.com \\ ${ }^{3}$ School of Mathematics and Statistics, Zhengzhou University, Henan-450001, China.
}

\section{Abstract:}

Objectives: To examine the effect of unequal gap spacing for flow past over three rectangular rods a fixed Re (Reynolds number) and Aspect Ratio (AR). Methodology: A two dimensional (2-D) numerical study of flow over three rectangular rods is conducted by using Single Relaxation Time Lattice Boltzmann Method (SRT-LBM). The gap spacing (g) between the rods is varied from 0.5 to 7 with fixed Reynolds number i.e., $R e=150\left(R e=. U_{\infty} d / v\right.$., where $U_{\infty}$ is uniform velocity, $d$ is size of an object and $v$ is the kinematic viscosity) and aspect ratio $A R=0.25$ (where, $A R=l / w, l$ is the length and $w$ is the width of the rods). Different flow structures, power spectra analysis of lift coefficients and force statistics are studied for this analysis at small, moderate and large gap spacing. Findings: The present study revealed that the gap spacing between the rods has a great impact on the flow behaviour. Therefore, different flow patterns are observed and some of them are outlined as, i). Shear layer reattachment (SLR), ii). Semi developed single bluff body (SDSBB), iii). Fully developed vortex shedding (FDVS), iv). Semi developed irregular vortex shedding, v) Fully generated two row single bluff body, vi). Semi developed irregular single bluff body (SDISBB) and vii). Critical flow (CF) patterns. The critical flow behaviour is observed at the gap spacing $\left(g_{1}, g_{2}\right)=(5,2.5)$, (here, $g_{1}$ is the gap between upstream rods to middle one and $g_{2}$ is the gap between middle rods to downstream rectangular rod). In force statistics analysis, the values of $\mathrm{C}_{\mathrm{dmean}}$ and Strouhal number are computed at different spacing ratios. The value of mean drags coefficients for all three rodsat $g_{1}=3$ is approximately same, but at $g_{1}=1$ and 5 , the maximum value of $\mathrm{C}_{\text {dmean }}$ is observed for $\mathrm{C} 2$ as compared to $\mathrm{C} 1$ and $\mathrm{C} 3$, that is 6.91 . Furthermore, Strouhal number values are much less than the single rod (SR) value for all three chosen small, moderate and large gap spacing values. The maximum value of $S_{t}$ is examined at $\left(g_{1}, g_{2}\right)=(1,1)$ i.e 0.19 .

Keywords: Drag Coefficient; Rectangular Rods, Single Relaxation Time Lattice Boltzmann Method, Vortex Shedding Suppression

\section{Nomenclature}

$\begin{array}{ll}\mathrm{Cd} & \text { Drag coefficient } \\ \mathrm{Cl} & \text { Lift coefficient } \\ \mathrm{C}_{\mathrm{dmean}} & \text { Mean drag coefficient } \\ \mathrm{C}_{\mathrm{drms}} & \text { Root-mean-square value of drag coefficient } \\ \mathrm{c} & \text { Lattice speed } \\ \mathrm{c}_{\mathrm{s}} & \text { Speed of sound } \\ \mathbf{e}_{\mathrm{i}} & \text { Velocities direction } \\ \mathrm{f}_{\mathrm{s}} & \text { Vortex shedding frequency } \\ \mathrm{h}_{\mathrm{i}} & \text { Particle distribution function } \\ \mathrm{h}_{\mathrm{i}} \text { (eq) } & \text { Equilibrium distribution function } \\ \mathrm{g} & \text { Gap spacing }\end{array}$

$\mathrm{H} \quad$ Height of the computational domain

$\mathrm{L}_{\mathrm{u}} \quad$ Upstream position

$\mathrm{L}_{\mathrm{d}} \quad$ Downstream position

Q $\quad$ Number of particles

Re Reynolds number

$S_{t} \quad$ Strouhal number

$\mathrm{U}_{\infty} \quad$ Uniform inflow velocity

\section{Greek Symbols}

$v \quad$ Kinematic viscosity

$\rho \quad$ Fluid density

$\omega_{i} \quad$ Weighting coefficients

$\tau$ Relaxation time

*Author for correspondence 


\section{Introduction}

Fluid flow around more than one objects have attracted much attention in recent times due to its immense contribution in the field of engineering and industries. Its applications are in off-shore structures, heat exchangers, sea-crossing bridges, micro-electro-mechanical devices (MEMS) and other solar equipment. When flow interacts with these structures, it gives rise to complex evolvement of the vortices that ultimately produced drag and lift forces, that create noise and flow induced vibration. As a result damaging of structures occurs. In this regard, many experimental and numerical works has been done to overcome these noises using square/circular rods. These rods are placed in line, side-by-side and in staggered arrangements. As compared to circular or square rods, less work has been done on the rectangular rods. Rectangular rods behave differently as compared to circular rods. In case of rectangular rods, due to large circulation regions, broad wakes appear and separation point is fixed at the front leading edge. Many experimental and numerical studies are available in literature for flow past over a circular, square and rectangular objects. In [1] conducted an experimental study on the vortex shedding frequency of the various single rectangular rods at $70 \leq \operatorname{Re} \leq 20,000$. The experiment revealed that the vortices reattached twice on the wall of rod at $\operatorname{Re}=150-800$ as the length to width ratio raises beyond 2. An experimental study of large eddy simulation (LES) for flow past rectangular-section performed by [2] revealed different flow characteristics at side ratio $0.4 \mathrm{~d}$ to $4 \mathrm{~d}$ and $\mathrm{Re}=10^{5}$. It is observed that flow showed local instabilities, which can lead to global instabilities. In [3] conducted 2-D experimental study of the turbulence behavior near wake flow behind the rectangular rod using hot wire anemometer. At different aspect ratio (AR), time averaged and fluctuating velocities are measured. As the aspect ratio increases, turbulence decreases at near wake flow. In [4] numerically investigated the flow around a rectangular rod using Finite Difference Method (FDM) at the range of parameters, $100 \leq \operatorname{Re} \leq 120$ and $0.4 \mathrm{~d} \leq \mathrm{AR} \leq 8 \mathrm{~d}$. The critical flow pattern was observed at $\mathrm{AR}=2.8 \mathrm{~d}$ and $\mathrm{Re}=500-1200$. Similar numerical simulation was performed by [5], in which laminar and turbulent flow around a rectangular rod was studied. As the side ratio changes from $2.1 \mathrm{~d}$ to $6 \mathrm{~d}$, vortices alternatively reattached at downstream location of channel. In [6] numerically investigated the flow around a rectangular rod at different aspect ratios at $\operatorname{Re}=400-30,000$. A change in the drag coefficients was found at $A R=0-1$. The maximum value of the $C_{d m e a n}$ was observed at $A R=0.6 \mathrm{~d}$. In [7] performed numerical investigation of unsteady flow around rectangular rod at low Reynolds numbers using Third-order and second-order Crank-Nicolson scheme by taking $A R=0.4 d$ to $8 d$ and $R e=100-200$. Change in Strouhal number $\left(\mathrm{S}_{\mathrm{t}}\right)$ was observed at $\mathrm{Re}=200$ and $\mathrm{AR}=$ $0.5 \mathrm{~d}$. In [8] performed three dimensional (3-D) unsteady flow around a rectangular rod at $\mathrm{AR}=5: 1, \mathrm{Re}=26,400$, low Mach number $\left(M_{\infty}=0.1\right)$ and zero degree of attack. Detached Eddy Simulation (DES) was used for turbulence modelling. Result evaluated using hybrid mesh, attracted much attention toward the span-wise extension of the computational domain. To discretize the inviscid fluxes in governing equation, second order Central Difference Scheme (CDS) was applied. Results followed from DES exhibited that hybrid method is well applicable to all problems in which large value of Re is involved. In [9] numerically investigated the flow around rectangular rod using Incompressible lattice Boltzmann method (ILBM) with $A R=0.15 d-4 d$ and $R e=100-250$. Drastic change in the drag coefficients was observed at $A R=0.15 \mathrm{~d}-2 \mathrm{~d}$. Zhu Huang et al., [10] numerically simulated the flow around two rectangular rods in tandem at $\mathrm{Re}=200$ with $\mathrm{AR}=1: 2$ using Multi-Domain Pseudo-Spectral method (MPS). Flow is assumed to be laminar and uniform. They concluded that at the critical gap spacing of $g=5$, drag coefficients approached its maximum value. Also, at the critical gap spacing vortices behind the two rods were shedding in an anti-phase mode. In[11] conducted an experimental study on the flow around two circular rods arranged tandemly. He noticed critical gap spacing at $\mathrm{g}=$ 3.5 with $\mathrm{Re}=22000$. In [12] experimentally exhibited flow around two inline circular rods and examined critical $g$ at 3.5 with negative drag coefficients. In [13] experimentally study the flow around three inline circular rod sat 10900 $\leq \mathrm{Re} \leq 39200$. It was examined that the drag coefficient is high for upstream rod as compared to downstream and middle stream rod has less value of the drag coefficient as compared to other two rods. In [14] numerically simulated the flow around two and three circular rods arranged inline at $\operatorname{Re}=100$ and 200. For the inline rods, at $\mathrm{Re}=100$ steady wake patterns observed and sparse Karman street formed at $\mathrm{Re}=200$ for $\mathrm{g}=2$. Islam et al. [15] numerically examined the flow around three square rods with varied gap spacing and critical flow pattern was observed at $g=3$. All above mentioned studies revealed that less much concentration is paid for flow past three or 
more than three rectangular rods at various gap spacing to study flow structure mechanism. Therefore, in present study, we examined the effect of unequal gap spacing for flow past over three rectangular rods a fixed Re and AR.

This paper is arranged as follows. Introduction is discussed in Section 1, numerical method is described in Section 2, Section 3 consists of problem statement, code validation study, effect of computational domain and impact of uniform inflow velocity will be discussed in Section 4. Results and discussions will be presented in Section 5. Finally, conclusion is drawn in Section 6.

\section{Lattice Boltzmann Method}

The numerical technique Single Relaxation TimeLattice Boltzmann Method (SRT-LBM) for the particle distribution function $\mathrm{h}$ is applied to solve present problem. The lattice structure D2Q9, in which nine particles are involved is used here. The general form of LBE equation is given as

$$
h_{i}\left(x+e_{i}, t+1\right)-h_{i}(x, t)=-\frac{\left[h_{i}(x, t)-h_{i}^{(e q)}(x, t)\right]}{\tau}
$$

where $h_{i}$ is the particle distribution function and $h_{i}{ }^{(e q)}$ is the particle equilibrium distribution function, $\mathbf{e}_{\mathrm{i}}$ are the direction vectors and $\mathbf{x}$ is the position vector.The equilibrium distribution function is calculated as (WolfGladow [16])

$$
\mathrm{h}_{\mathrm{i}}^{(\mathrm{eq})}=\rho \omega_{\mathrm{i}}\left(1+3\left(\mathrm{e}_{\mathrm{i}} \cdot \mathrm{u}\right)+4.5\left(\mathrm{e}_{\mathrm{i}} \cdot \mathrm{u}\right)^{2}-1.5 \mathrm{u}^{2}\right)
$$

The kinematic viscosity is to be calculated through the relation $v=\frac{2 \tau-1}{6}$. Eq (1) can be solved through BGK approximation(Bhatnagar-Gross-Krook (BGK) [17]) i.e. through streaming and collision steps. During collision mass and momentum are conserved. In streaming particle at each node moved to its neighboring nodes at respective velocities(Wolf-Gladow [16]).

$$
h_{i}^{*}(x, t)=h_{i}(x, t)-\left[\frac{h_{i}(x, t)-h_{i}^{*}(x, t)}{\tau}\right]
$$

Where $h_{i}^{*}$ are the intermediate function.

$$
h_{i}\left(x+e_{i}, t+1\right)=h_{i}^{*}(x, t)
$$

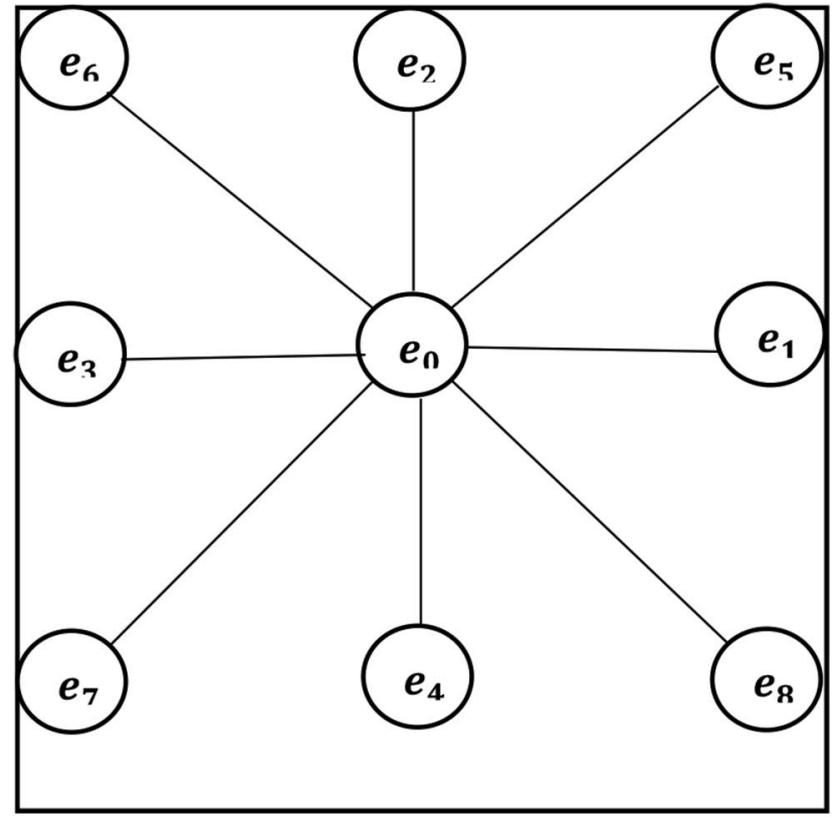

Figure 1. D2Q9 particles velocity model.

The density $(\rho)$, velocity (u) and pressure (p) at each computational node are calculated as

$$
\begin{array}{cc}
\rho=\sum g_{i} & i=0, \ldots, 8 \\
\boldsymbol{u}=\frac{1}{\rho} \sum g_{i} \boldsymbol{e}_{i} & i=1, \ldots, 8 \\
p=\rho c_{s}{ }^{2} &
\end{array}
$$

where $c_{s}$ is the speed of sound $(=1 / \sqrt{3})$.

\section{Problem Description and Boundary Conditions}

A two-dimensional (2-D) numerical study constitutes of three rectangular rods of width 'w'and diameter'd' between 2-D plane channel is carried out with upstream distance $\mathrm{L}_{\mathrm{u}}=6 \mathrm{~d}$, downstream distance $\mathrm{L}_{\mathrm{d}}=25 \mathrm{~d}$ and the height of the computational domain $\mathrm{H}=11 \mathrm{~d}$. The vital parameter is spacing ratio between the rods and its value varies from $g=0.5$ to 7 with fixed $\mathrm{Re}=150$ and $\mathrm{AR}=0.25 . \mathrm{C} 1, \mathrm{C} 2$, C3 are upstream, downstream, and middle stream rods, respectively. The uniform inflow velocity $\left(\mathbf{u}=\mathrm{U}_{\infty}, \mathbf{v}=0\right)$ is applied at the inlet and convective boundary condition $(\partial \mathbf{u} / \partial \mathbf{x}=\partial \mathbf{v} / \partial \mathbf{x}=0)$ is applied at the outlet of the channel. The no-slip $(\mathbf{u}=\mathbf{v}=0)$ boundary conditions are applied 

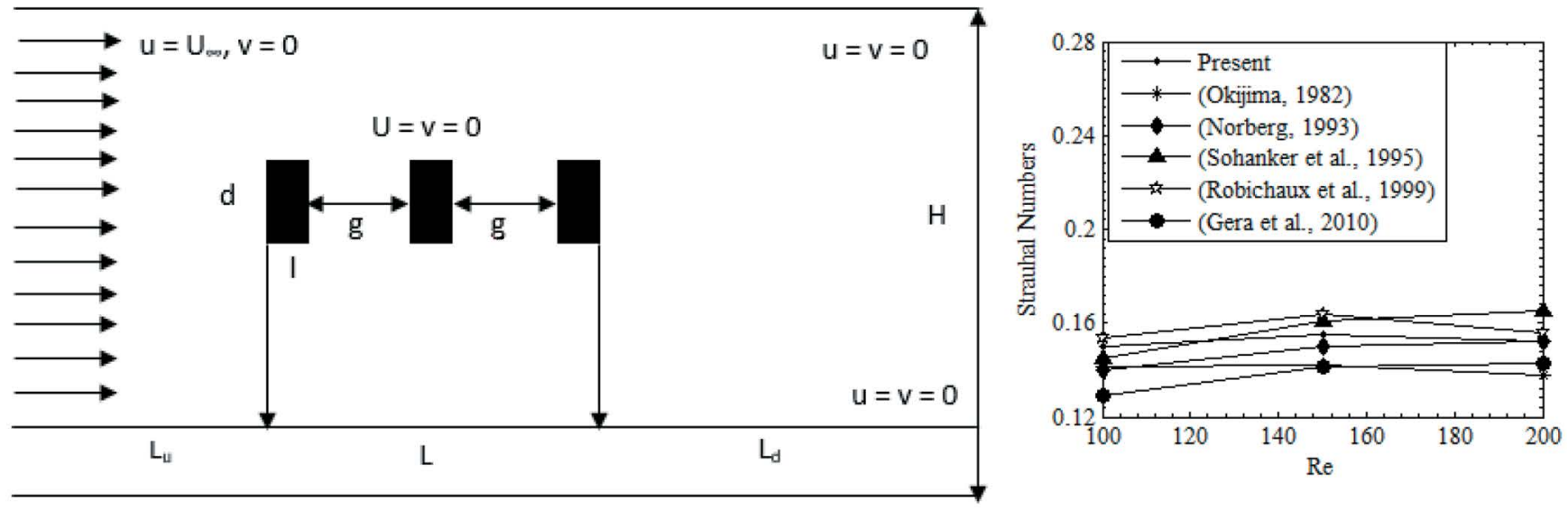

Fig. 2: A schematic diagram for flow past three rectangular rods

at the surface of the rod, as well on the top and bottom wall of the channel (Cheng et al. [18]). The fluid forces are calculated using momentum exchange method (Dazchi et al. [19]).

\section{Code Validation Study, Effect of Computational Domain and Impact of Uniform Inflow Velocity}

\subsection{Code Validation Study}

To check the validity of present code, the physical parameters such as $\mathrm{C}_{\text {dmean }}$ and $\mathrm{S}_{\mathrm{t}}$ of single rectangular rod at $\mathrm{Re}=80-200$ are calculated and compared with the values of $\mathrm{C}_{\mathrm{dmean}}$ and $\mathrm{S}_{\mathrm{t}}$ are available in literature both experimentally and numerically. The values of $\mathrm{C}_{\text {dmean }}$ at $\operatorname{Re}=150$ found good agreement with the numerical work of Norberg [6] and experimental work of Okajima [1]. While, the values of the Strouhal number at $\mathrm{Re}=140$ 160 found excellent agreement with experimental and numerical work of Okajima [1], Norberg [6], Robichaux [20], and Gera [21] etc, that assure the validity of present code, (see Figure 3).

\subsection{Effect of Computational Domain}

Different numerical simulations have been performed for flow around single rectangle rod at different values of $\mathrm{L}_{\mathrm{u}}$, $\mathrm{L}_{\mathrm{d}}$ and $\mathrm{H}$. The corresponding results obtained in the form of mean drag force and Strouhal number. First, varying $L_{u}$ from $5 d$ to $7 d$, keeping $L_{d}$ and $H$ fixed we noticed that values of the mean drag forces decreases while values

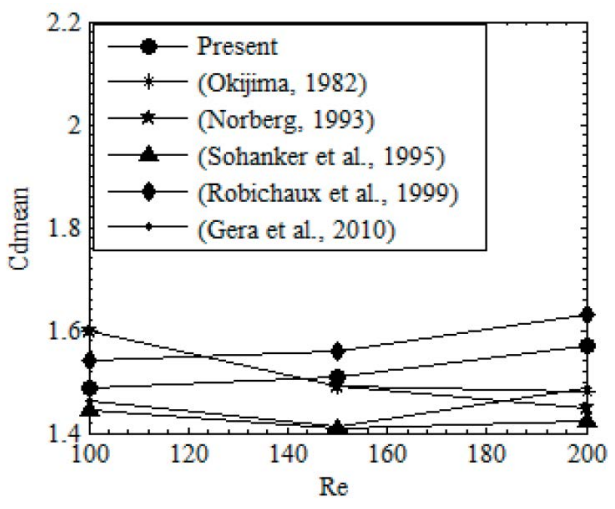

Figure 3. Comparison of $\mathrm{S}_{\mathrm{t}}$ and $\mathrm{C}_{\mathrm{dmean}}$ at different Reynolds number.

of the $\mathrm{S}_{\mathrm{t}}$ also showed deterioration. In comparison with literature, $S_{t}$ showed good experimental and numerical agreement with Norberg [6] and Sohanker et al. [7]. Secondly, we kept upstream distance and height of the computational domain constant while changing downstream location from $20 \mathrm{~d}$ to $30 \mathrm{~d}$. We observed that mean drag forces showed minor difference in its values and Strouhal number remain the same. Their values are in good agreement with the Norberg, [6] and Sohanker et al. [7]. In third case, we kept $\mathrm{L}_{\mathrm{u}}$ and $\mathrm{L}_{\mathrm{d}}$ uniform and only made change in $\mathrm{H}$ from $9 \mathrm{~d}$ to $12 \mathrm{~d}$. We came to know that $\mathrm{C}_{\text {dmean }}$ and $\mathrm{S}_{\mathrm{t}}$ both have a decreasing behavior. Also, found great conformation with the work of Norberg [6] and Sohanker et al. [7]. Therefore, to proceed present work, we selected $\mathrm{L}_{\mathrm{u}=} 6 \mathrm{~d}, \mathrm{~L}_{\mathrm{d}=} 25 \mathrm{~d}$ and $\mathrm{H}=11 \mathrm{~d}$, see Table 1 .

\subsection{Impact of Uniform Inflow Velocity}

The uniform inflow velocity played a significant role in the flow structure phenomena. It has the power to delay 
Table 1. Computational domain independence study at $\operatorname{Re}=150$.

\begin{tabular}{|l|c|c|}
\hline \multicolumn{1}{|c|}{ Cases } & $\mathrm{C}_{\text {dmean }}$ & $\mathbf{S}_{\mathrm{t}}$ \\
\hline Norberg [6] & - & 0.150 \\
Okajima [1] & 1.492 & 0.141 \\
Gera et al. [16] & 1.411 & 0.141 \\
Sohanker et al. [7] & 1.408 & 0.161 \\
\hline \multicolumn{2}{|c|}{ Upstream Location $\left(\mathrm{L}_{\mathrm{u}}\right)$} \\
\hline $\mathrm{L}_{\mathrm{u}}=5 \mathrm{~d} ; \mathrm{L}_{\mathrm{d}}=25 \mathrm{~d} ; \mathrm{H}=11 \mathrm{~d}$ & 1.5410 & 0.1571 \\
$\mathrm{~L}_{\mathrm{u}}=6 \mathrm{~d} ; \mathrm{L}_{\mathrm{d}}=25 \mathrm{~d} ; \mathrm{H}=11 \mathrm{~d}$ & 1.5390 & 0.1560 \\
$\mathrm{~L}_{\mathrm{u}}=7 \mathrm{~d} ; \mathrm{L}_{\mathrm{d}}=25 \mathrm{~d} ; \mathrm{H}=11 \mathrm{~d}$ & 1.5320 & 0.1520 \\
\hline \multicolumn{3}{|c|}{ Downstream Location $\left(\mathrm{L}_{\mathrm{d}}\right)$} \\
\hline $\mathrm{L}_{\mathrm{u}}=6 \mathrm{~d} ; \mathrm{L}_{\mathrm{d}}=20 \mathrm{~d} ; \mathrm{H}=11 \mathrm{~d}$ & 1.5320 & 0.1571 \\
$\mathrm{~L}_{\mathrm{u}}=6 \mathrm{~d} ; \mathrm{L}_{\mathrm{d}}=25 \mathrm{~d} ; \mathrm{H}=11 \mathrm{~d}$ & 1.5301 & 0.1571 \\
$\mathrm{~L}_{\mathrm{u}}=6 \mathrm{~d} ; \mathrm{L}_{\mathrm{d}}=30 \mathrm{~d} ; \mathrm{H}=11 \mathrm{~d}$ & 1.5210 & 0.1571 \\
\hline \multicolumn{2}{|c|}{ Height of Computational Domain $(\mathrm{H})$} \\
\hline $\mathrm{L}_{\mathrm{u}}=6 \mathrm{~d} ; \mathrm{L}_{\mathrm{d}}=25 \mathrm{~d} ; \mathrm{H}=9 \mathrm{~d}$ & 1.5440 & 0.1615 \\
$\mathrm{~L}_{\mathrm{u}}=6 \mathrm{~d} ; \mathrm{L}_{\mathrm{d}}=25 \mathrm{~d} ; \mathrm{H}=11$ & 1.5420 & 0.1571 \\
$\mathrm{~L}_{\mathrm{u}}=6 \mathrm{~d} ; \mathrm{L}_{\mathrm{d}}=25 \mathrm{~d} ; \mathrm{H}=12 \mathrm{~d}$ & 1.5401 & 0.1527 \\
\hline
\end{tabular}

or fast the vortex shedding phenomena. It can be easily noticed that, at $\mathrm{U}_{\infty}=0.0268$ and $\mathrm{U}_{\infty}=0.031$ the vortex shedding is very delayed as compared to other values of the uniform inflow velocity. At $\mathrm{U}_{\infty}=0.05834$ the vortex shedding phenomena is very fast and easily disturbs the flow. So, selected $\mathrm{U}_{\infty}=0.04385$ to solve this problem.
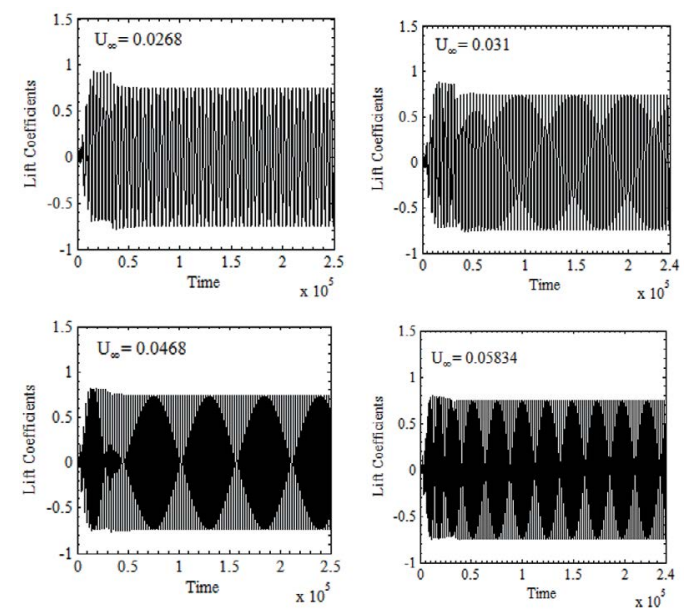

Figure 4. Comparison of different values of uniform inflow velocity.

\section{Results and Discussions}

To study the flow behavior over rectangular rods at unequal gap spacing by taking $g_{1=} 1,3$ and 5 and $g_{2}=$ 0.5-7 with fixed $\mathrm{Re}=150$ and $\mathrm{AR}=0.25$, obtained results are classified into three different flow regimes, i) Small wake regime, ii) Moderate wake regime, iii) Large wake regime.

\subsection{Vorticity Contour Visualization}

\subsubsection{Small Wake Regime}

This flow regime consists of $\mathrm{g}_{1}=1$ and varying $\mathrm{g}_{2}$ from 0.5 to 7 . At small gap spacing when $g_{1}=1$ and $1 \leq g_{2} \leq 1.5$, if $g_{2}=1$ as soon as the flow interacts with the downstream rod $(\mathrm{C} 1)$, immediately gets attached to middle stream (C2) and upstream rod (C3) treating three as one rod. From the boundary of the C3, flow rolls up and generate vortices in an alternate fashion, we assign a name shear layer reattachment single bluff body (SLRSBB) flow pattern in (Figure 5(a)). This flow pattern is also observed experimentally by Abdollah et al. (2008) for $\operatorname{Re}=8600$ and 17400 and numerically by Shams et al. (2012) for $R e=100-250$ for different aspect ratios in case of single rectangular cylinder. Similar behavior is observed for $\mathrm{g}_{2}=1.5$ but number of vortices decreases quite sharply. At $g_{1}=1$ and $2 \leq g_{2} \leq 2.5$, the flow arrived at $\mathrm{C} 1$ and vortices are completely generated in first gap between $\mathrm{C} 1$ and $\mathrm{C} 2$, but partial generation of vortices take place in second gap spacing between $\mathrm{C} 2$ and $\mathrm{C} 3$. After that vortices detached from $\mathrm{C} 3$ and move in an alternate fashion. That is why, it named as semi generated single bluff body (SGSBB) flow pattern in (Figure 5(b,c)). By increasing the gap spacing, keeping $\mathrm{g}_{1}=1$ and $3 \leq g_{2} \leq 5$, showed pattern is slightly different from what we observed. So, the main difference is that vortices increase in numbers and due to increase in gap, complete form of shed vortices appear.

The power spectra analysis of lift coefficients for three rods with varying gap spacing are given in graphs of $\mathrm{S}_{\mathrm{t}}$. Here C1, C2 and C3 are Showing vortex shedding frequency of upstream, middle stream and upstream rod. For shear layer reattachment single bluff body and semi generated single bluff body, the sharp peaks of the Strouhal number has been observed. We also observed that by increasing gap spacing from $\mathrm{g}_{2}=1$ to 4 , middle stream and upstream rods affected quite badly. Some multi peaks has been observed. By comparing their magnitude, major distortion has been observed at C3as shown in (Figure 6(a-f)). The maximum magnitude of St obtained at $g_{1=} 1$ and $g_{2}=2.5$ for all three selected rods in small wake regime clear from Figure 6(f). 

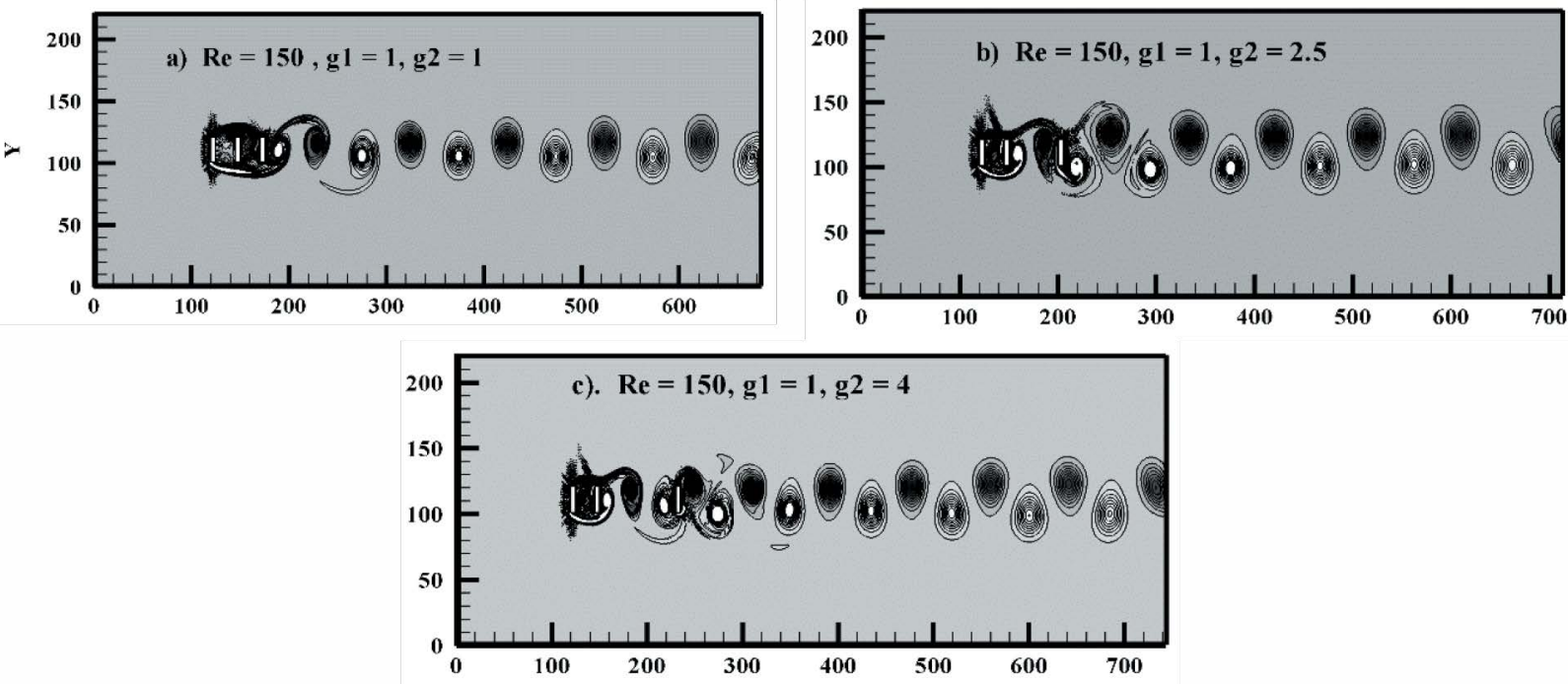

Figure 5. Vorticity contour visualization for (a) Shear layer reattachment single bluff body, (b, c) Semi developed single bluff body.
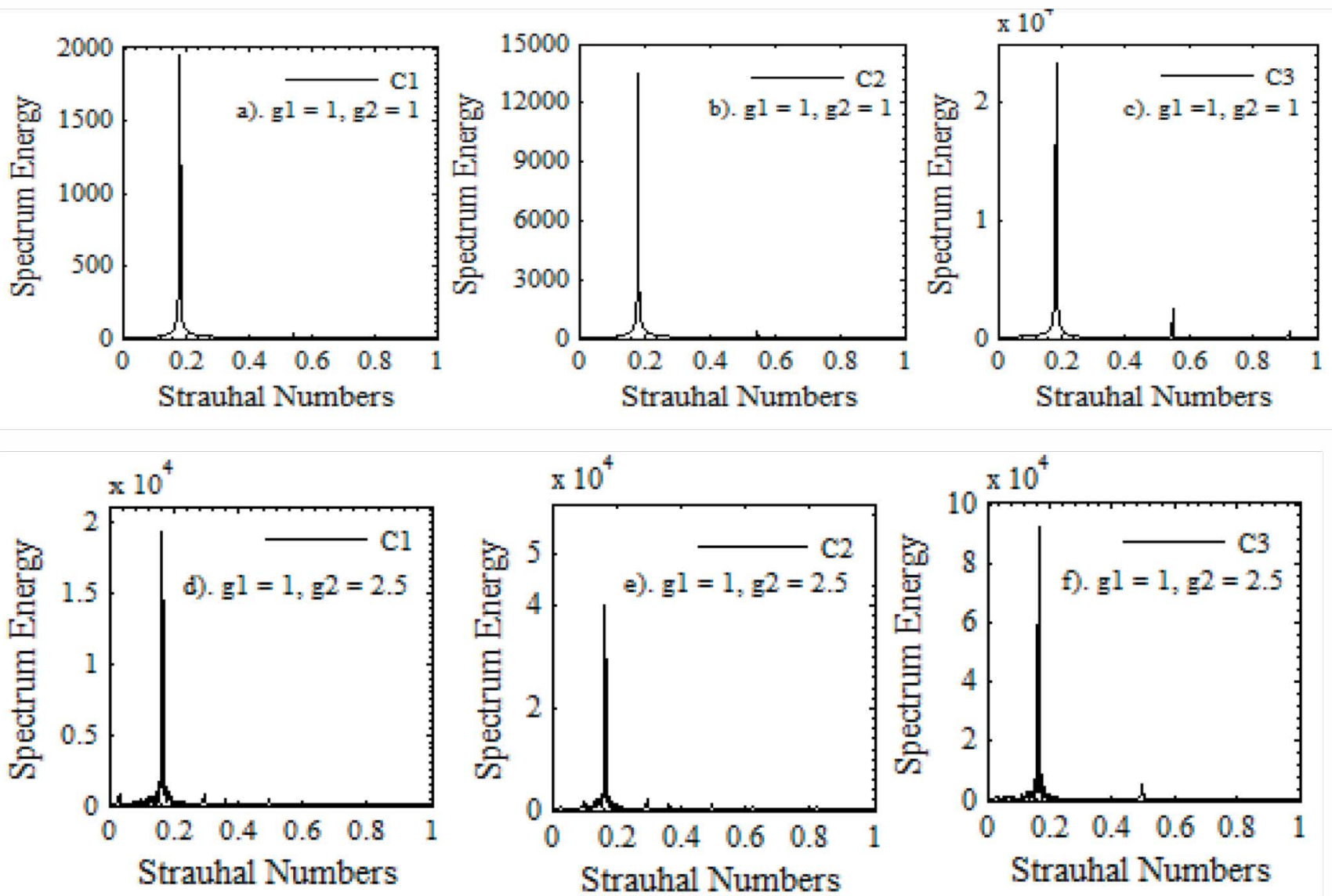

Figure 6(a-f). Power spectra analysis of (a-c) Shear layer reattachment single bluff body, (d-f) Semi developed single bluff body. 


\subsubsection{Moderate Wake Regime}

Due to enough spacing in first gap, at $\mathrm{g}_{1}=3$ and $\mathrm{g}_{2}=1$ vortices completely generated in first gap between $\mathrm{C} 1$ and C2. But partial generation of vortices takes place in second gap between $\mathrm{C} 2$ and $\mathrm{C} 3$. As soon as the vortices separated from C3, they immediately arranged themselves in an alternate way. Such behavior of flow is called as semi developed vortex shedding (SDIVS) flow pattern (see Figure 7(a)) with respect to second gap. By increasing gap spacing up-to some extent means $g_{2}=1.5$, flow pattern shows similarity with the semi developed vortex shedding flow pattern, but major change occurred at C3. Flow becomes unsteady or irregular as it passes through $\mathrm{C} 3$ for a little while. After wards continue its motion in a typical way means alternatively. (see Figure $7(\mathrm{~b})$ ). At $\mathrm{g}_{1}=3$ and $\mathrm{g}_{2}=2$ and 3, vortices fully formed in first and second gap. But abruptly changes its shape as its goes through C3. They transformed themselves immediately in to two parallel rows for a short while, later move alternatively. This flow pattern due to its detreating behavior called as fully generated two row single bluff body (FGTRSBB) flow pattern (Figure $7\left(\mathrm{c}, \mathrm{d}\right.$ )). At $\mathrm{g}_{1}=3$ and $\mathrm{g}_{2}=2.5,4$, 5 , the vortices completely generated in between the two gaps and may differ from each other by shape, size and in numbers. This flow pattern is categorized as fully developed vortex shedding (FDVS) flow pattern (Figure $7(\mathrm{e})$ ). The Power spectra analysis of moderate wake regime show that semi developed vortex shedding, sharp peak has been noted. But for semi developed irregular vortex shedding flow pattern, flow changes its behavior from steady to unsteady as it emerged through C3. That is why $\mathrm{C} 3$ in the graph has noisier and disrupt behavior (see Figure 8(c, f, I, l, o). But overall sharp peak has been clearly seen. For fully generated two row single bluff body flow pattern, power spectrum analysis observed some harmonic peaks (see Figure 8). Since the vortices
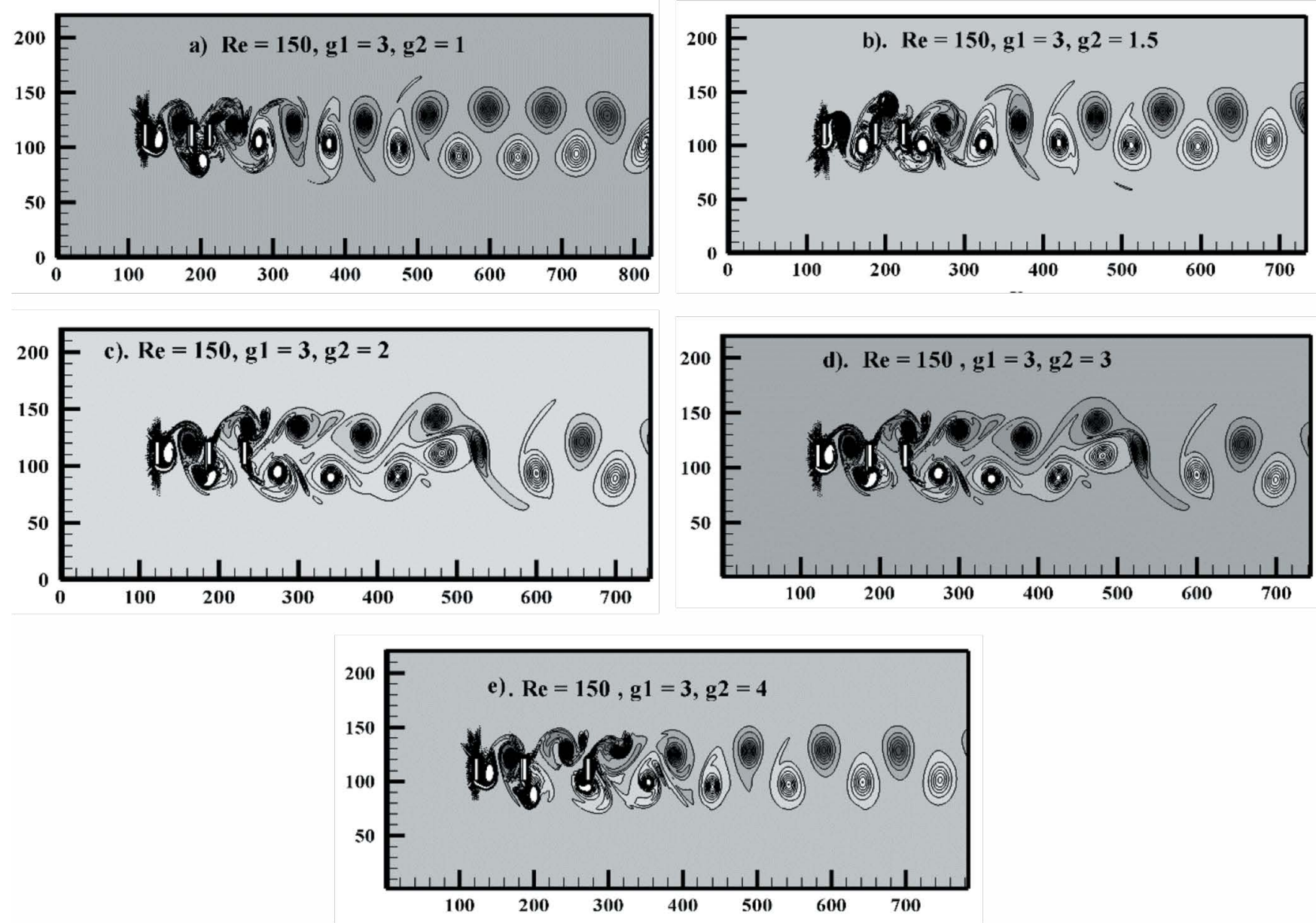

Figure 7. Vorticity contour visualization for ( $a, b)$ Semi developed irregular vortex shedding, (c, d) Fully developed two row single bluff body and (e) Fully developed vortex shedding. 

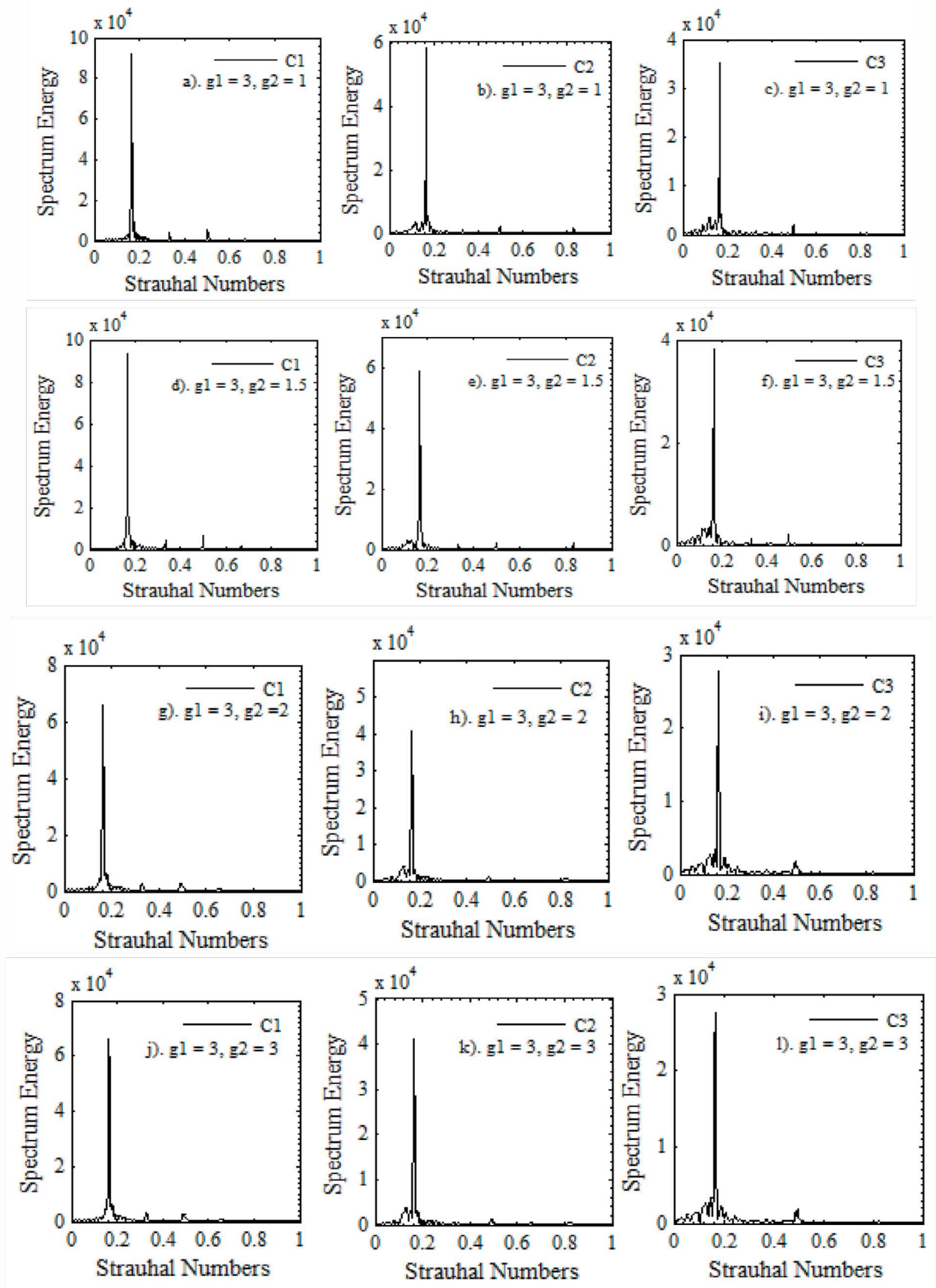

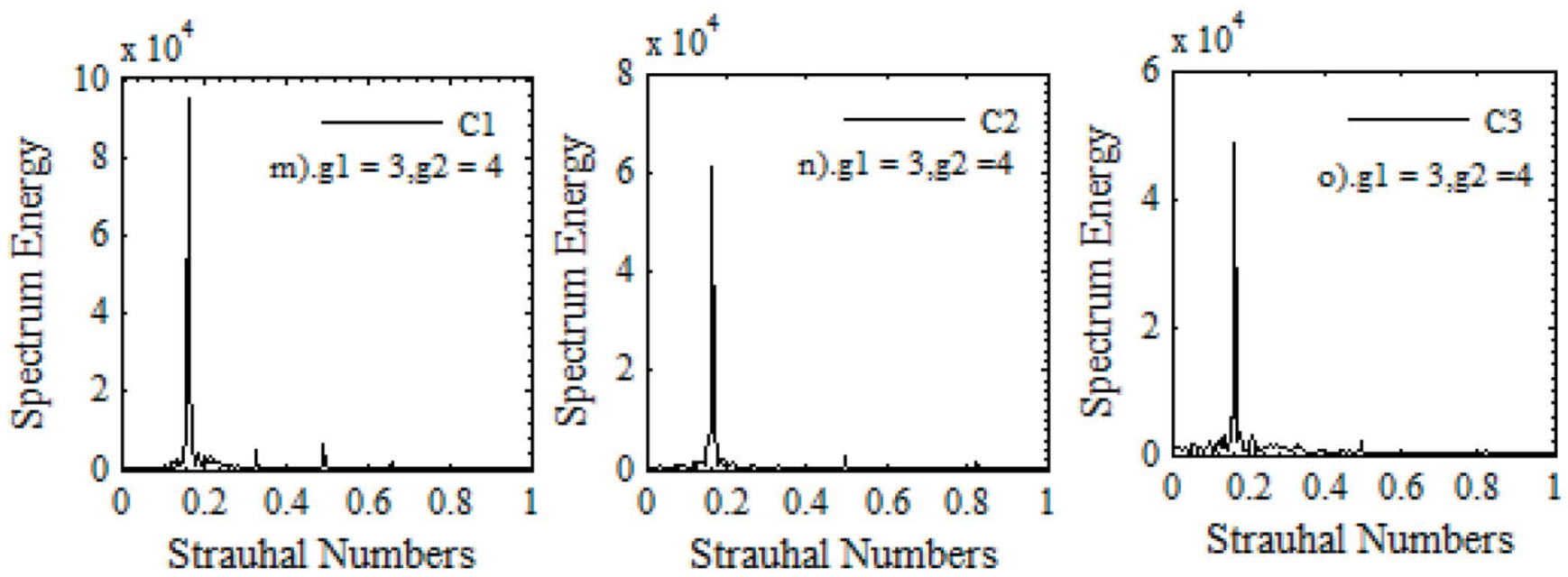

Figure 8(a-o). Power spectra analysis for (a, f) Semi developed irregular vortex shedding, (g- l) Fully developed two row single bluff body and (m-o) Fully developed vortex shedding.

fully generated through $\mathrm{C} 1$, so sharp peak for $\mathrm{C} 1$ is noted. Since C2 under gone complete vortex shedding with merging of vortices at downstream location, that why some secondary peaks with primary frequency has been observed. Initially two peaks have been noted, later converted themselves in to single sharp peak. Similarly, for fully developed two row single bluff body flow pattern, a strong distortion found at $\mathrm{C} 3$ because from the $\mathrm{C} 3$ flow strongly changed its behavior. For fully developed vortex shedding, power spectra analysis exhibited sharp peak but distortion has been there, due to change in strength, size and shape of the vortices, (see Figure $8(\mathrm{a}-\mathrm{o})$ ). In comparison of Strouhal number magnitude, for moderate wake regime, the maximum magnitude will be found for first cylinder at $g_{1}=3$ and $g_{2}=4$ for fully developed single bluff body flow.

\subsubsection{Large Wake Regime}

When $g_{1}=5$ and $1 \leq g_{2} \leq 1.5$, there is enough space, therefore, vortices formed completely. But in the second gap, due to small space they could not fully evolved. From $\mathrm{C}_{3}$ to onward, flow strongly became unsteady. This flow pattern is called semi developed irregular single bluff body (SDISBB) flow pattern in (Figure 9(a)). This flow pattern also has been observed for small wake regime. At $g_{2}=1.5$ similar pattern has been noted, but strength of vortices improved. At $g_{1}=5$ and $g_{2}=2.5$ and6, here two extremes of gap spacing have been observed. Flow became highly irregular as it passed from C3 and scattered in different direction. We called this pattern as critical flow (CF) pattern see (Figure 9(b)).Igarashi [24] experimentally investigated the critical gap spacing (critical flow pattern) with similar flow and force coefficients characteristics for flow past two tandem circular cylinders at $\mathrm{g}=3.5$ and $\mathrm{Re}$ $=2.2 \times 10^{4}$. Kim et al. [25] experimentally observed that $g$ $=2.5$ is the critical gap spacing for flow past two tandem square cylinders $(\mathrm{AR}=1)$. At $\mathrm{g}_{1}=5$ and $2 \leq g_{2} \leq 5$, flow showed almost similar behavior with some minor differences. At C3, flow became irregular for short period. Only size, shape and number of vortices changes. We assign a name as fully developed irregular single bluff body (FDVS) flow pattern as shown in (Figure 9(c)).This flow pattern also examined for moderate wake regime at $g_{2}=2.5,4,5$. The power spectrum analysis of large wake regime of different flow pattern showed distortion. Irregularities, multiple peaks and in some cases secondary peaks also observed. Multi-peaks represent the irregular behavior. While, secondary peaks and distortion enable us to conclude that critical flow pattern has been observed at certain points in (Figure 10(a-i)).

\subsection{Force Statistics}

To study the effect of unequal gap spacing, we calculated the physical parameters, such as $\mathrm{C}_{\text {dmean }}$ and Strouhal numbers against $g_{2}$ by fixing $g_{1}$. The change in the mean drag coefficients by keeping $g_{1}=1$ and $1 \leq g_{2} \leq 6$ for three rods, showed that at $\mathrm{g}_{1}=1$, the value of $\mathrm{Cd} 1$ synchronizes with the $\mathrm{C}_{\text {dmean }}$ of single rod (SC) as shown in (Figure 11(a)). At the end, showed slight change in behavior at g2 $=6$, having value 2 . 1035.The Cd2 showed sudden 

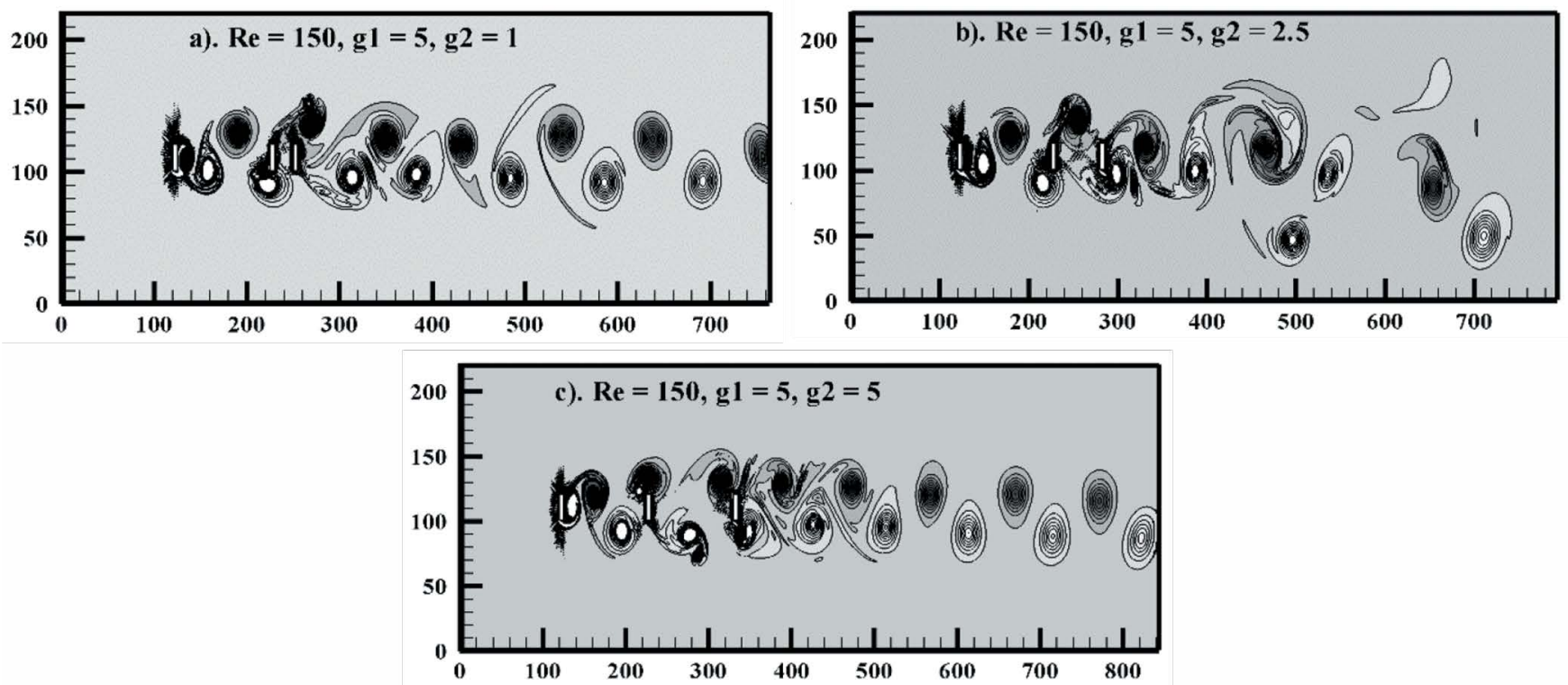

Figure 9. Vorticity contour visualization (a) Semi developed irregular single bluff body, (b) Critical flow pattern, (c) Fully developed vortex shedding
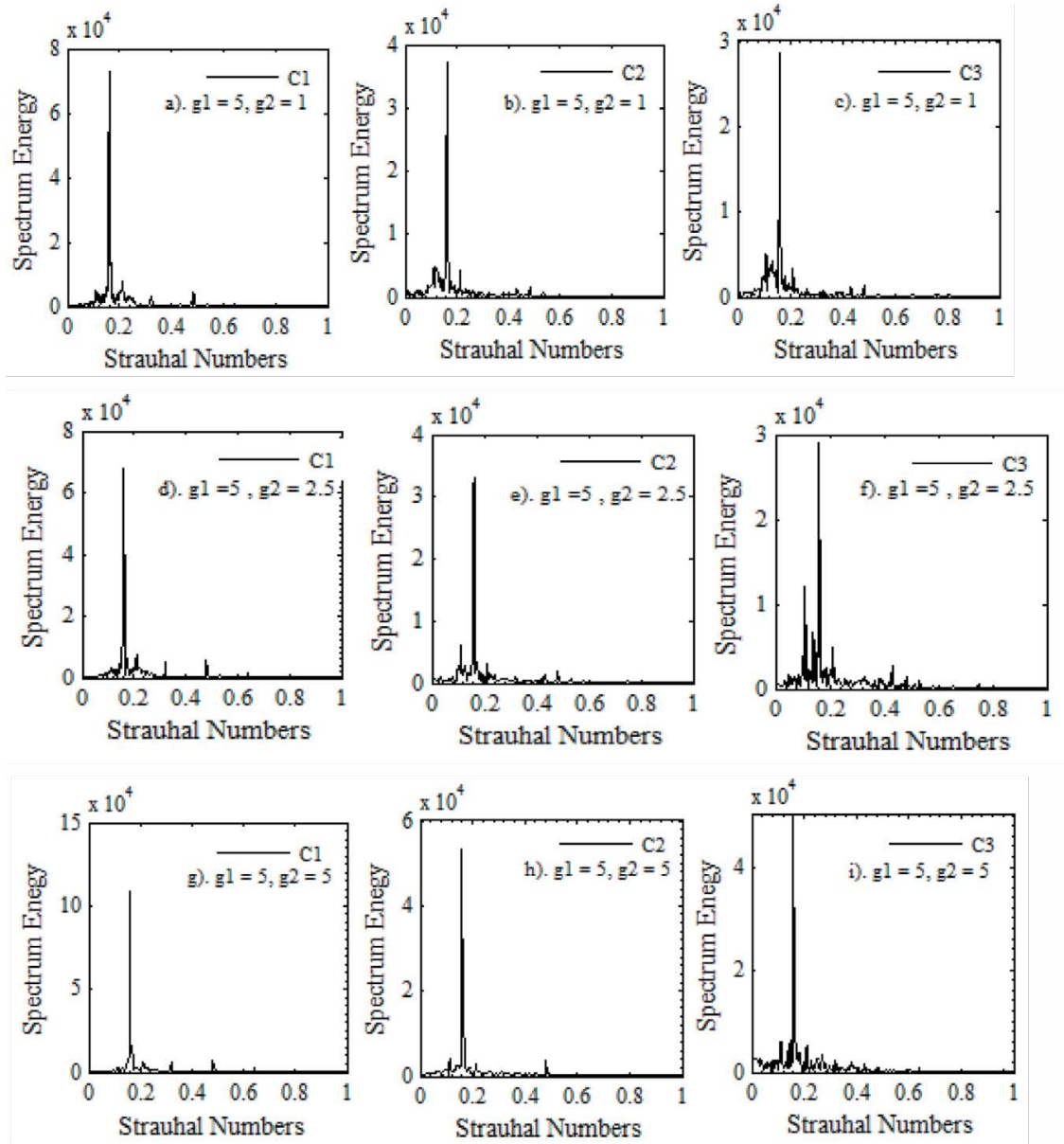

Figure 10(a-i). Power spectra analysis for (a-c) Semi developed irregular single bluff body, (d-f) Critical flow pattern, (g-i) Fully developed vortex shedding. 
jump at $\mathrm{g}_{2}=1.5$ and reached at its maximum value of 2.9841. Afterwards, $\mathrm{Cd} 2$ decreased quiet sharply and found minimum value at $\mathrm{g}_{2}=4$. Cd3 showed very unusual behavior as compared to $\mathrm{Cd} 1$ and $\mathrm{Cd} 2$. $\mathrm{Cd} 3$ reached its maximum value at $g_{2}=1.5$ that is 3.9064 . But as soon as it reached its maximum value, one can observe sudden
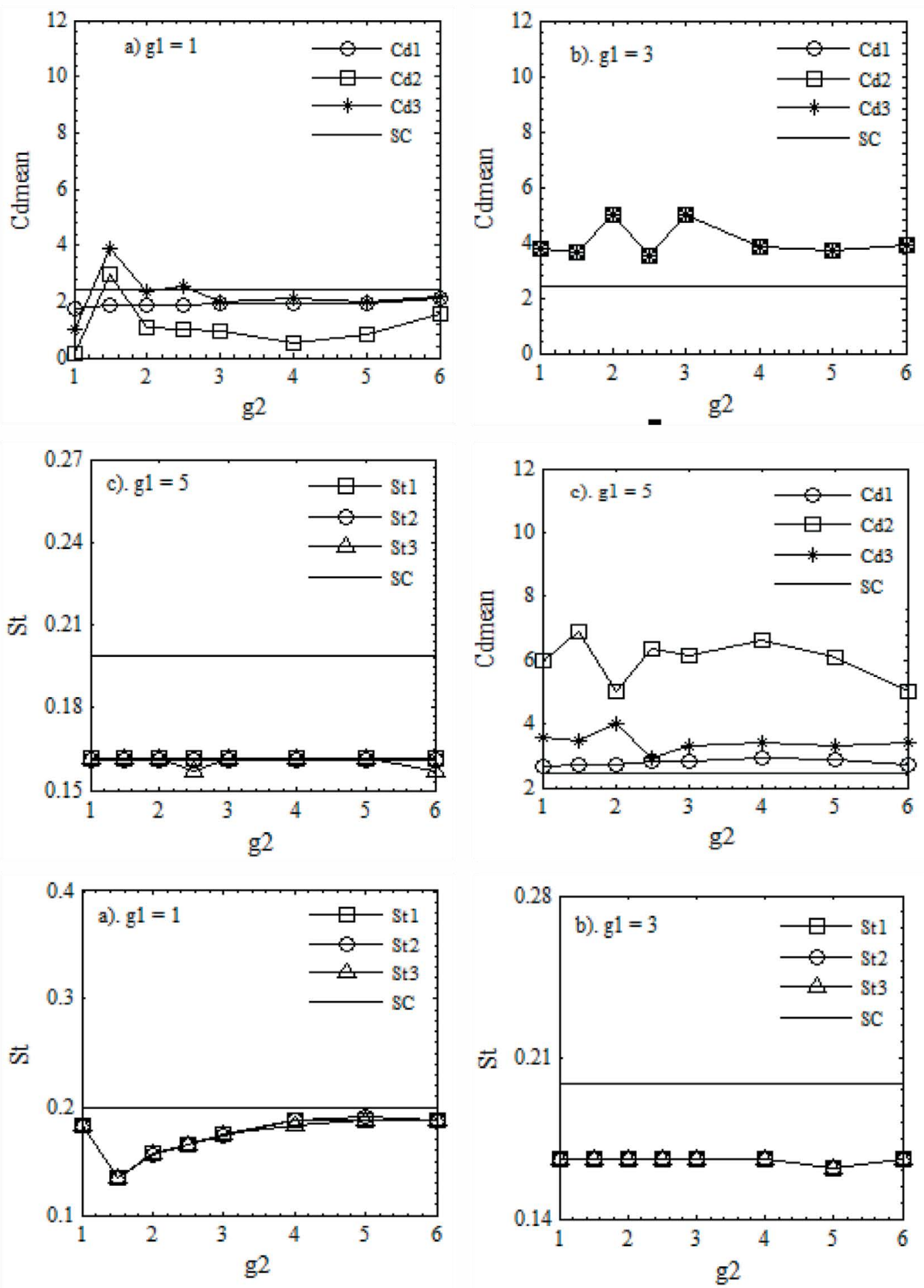

Figure 11(a-c). Comparison of $\mathrm{C}_{\text {dmean }}$ for (a) Small wake regime, (b) moderate wake regime and (c) large wake regime at different gap spacing. 
decline for the remaining gap spacing. In comparison with $\mathrm{SC}, \mathrm{Cd} 2$ and $\mathrm{Cd} 3$ have largest value at certain points. Other than this all Cdmean of C1, C2, C3 have smallest values than single rod. The change in the mean drag coefficients for $g_{1}=3$, revealed that all the three rods have some sort of overlapping of the values of the $\mathrm{C}_{\text {dmean }}$ that is clearly observable in the graph. Largest value of the $\mathrm{C}_{\mathrm{dmean}}$ has been noted twice i.e. $\left(g_{1}, g_{2}\right)=(3,2)$ and $\left(g_{1}, g_{2}\right)=(3$, 3 ) and smallest value has been observed at $g_{2}=2.5$. In comparison with single rod, all the values of $\mathrm{C}_{\text {dmean }}$ of three rectangular rods went through major decline as shown in (Figure 11(b)). From the graph of mean drag coefficients of $g_{1}=5$, one can notice that drag coefficients found its maximum value at $g_{2}=1.5$ and it is approximately 6.8985 for $\mathrm{C} 2$. It is because due to sufficient gap spacing. effect of $\mathrm{C} 1$ on $\mathrm{C} 2$ reduces After that, declined in the values is quite sharp, but still larger than the SC. While C1 and C2 have smallest value of $\mathrm{C}_{\text {dmean }}$ but still much larger than the single rod in (Figure 11(c)).The comparison of the St with single rod at $\mathrm{g}_{1}=1$, exhibited that the $\mathrm{S}_{\mathrm{t}}$ of $\mathrm{C} 1$, C2and C3 have small values with minor differences in between them. But all the values of Strouhal number for three rods are less than the single rod (see Figure 12(a)). While, at $g_{1}$ $=3$, all the $S_{t}$ of rods have special kind of synchronization in the values. Still there are less than the $S_{t}$ of the SC shown in Figure 12(b). When we further increase the gap spacing at $g_{1}=5$, we got that all the $S_{t}$ of three rods are so small than the $S_{t}$ of single rod as shown in Figure 12(c). All the values of the $\mathrm{S}_{\mathrm{t}}$ of $\mathrm{C} 1, \mathrm{C} 2$ and $\mathrm{C} 3$ coincide with each other with minor differences.

\section{Conclusions}

A numerical investigation of the flow around three rectangular rods using SRT-LBM with $\mathrm{Re}=150, \mathrm{AR}=0.25$ and varying the gap spacing from $g_{1}=1,3$ and 5 and $g_{2}$ $=0.5$ to 7 revealed different results. Some are outlines as

- Seven different flow patterns has been observed. i). Shear layer reattachment single bluff body, ii). Semi developed single bluff body, iii). Fully developed vortex shedding, iv). Semi developed irregular vortex shedding, v) Fully generated two row single bluff body, vi). Semi developed irregular single bluff body, vii). Critical flow for small, moderate and large wake regime.

- The critical flow pattern has been observed when $\left(\mathrm{g}_{1}\right.$, $\left.\mathrm{g}_{2}\right)=(5,2.5)$ and $\left(\mathrm{g}_{1}, \mathrm{~g}_{2}\right)=(5,6)$, due to sufficiently large gap spacing flow is highly unsteady and become critical.

- The power spectra analysis showed that by increasing the gap spacing flow becomes unsteady and irregular, that is why multipeak has been observed. The amplitude of the values of the Strouhal number varied with the increase in the gap spacing. At critical flow, Strouhal number has primary and secondary peaks which depicts the unsteadiness in the flow.

- The change in the mean drag coefficient showed that maximum value of $\mathrm{C}_{\text {dmean }}$ is observed at $\left(\mathrm{g}_{1}, \mathrm{~g}_{2}\right)=(1,1.5)$ that is 6.9 and after that showed $\mathrm{C}_{\text {dmean }}$ decreases at the remaining gap spacing for small wake region. While at $\mathrm{g}_{1}=3$, mixed trend of the values of the $\mathrm{C}_{\text {dmean }}$ with varying gap spacing has been observed. When $\mathrm{g}_{1}=$ 5 , all the values of the $C_{\text {dmean }}$ showed increasing and decreasing behavior and larger than the single rod value.

- All the values of the Strouhal numbers are synchronized with each other but all are less than the Strouhal numbers of the single rods for all three chosen small moderate and large gap spacing. The maximum value of $S t$ is examined at $\left(g_{1}, g_{2}\right)=(1,1)$ i.e., 0.19 .

\section{References}

1. Okajima, A.:Strouhal number of rectangular rod. J. Fluid Mech. 123; 379-398(1982). https://doi.org/10.1017/ S0022112082003115.

2. Sohanker, A.: Large eddy simulation of flow past rectangular-section rods: Side ratio effects. J. Wind Eng. Ind. Aerodyn. 96(5); 640-655 (2008). https://doi.org/10.1016/j. jweia.2008.02.009.

3. Shadaram, A.; Fard, M. A.; Rostamy, N.: Experimental study of near wake flow behind a rectangular rod. J. Applied Sci. 5(8): 917-926 (2008). https://doi.org/10.3844/ ajassp.2008.917.926.

4. Okajima, A.; Nagahisa, T.;Rokugou, A.: A Numerical analysis of flow around rectangular rods. JSME Int. J. Ser. 2. Fluids engineering, heat transfer, power, combustion, thermophysical properties. 33(4): 702-711 (1990). https://doi. org/10.1299/jsmeb1988.33.4_702.

5. Okajima, A.; Ueno, H.; Sakai, H.: Numerical simulation of laminar and turbulent flows around rectangular rods. Int. J. Num. method in fluids.15(9): 999-1012 (1992). https://doi. org/10.1002/fld.1650150906.

6. Norberg, C.: Flow around rectangular rods: pressure forces and wake frequencies. J. Wind Eng. and Ind. Aerodyn. 49(1-3): 187-196 (1993). https://doi.org/10.1016/01676105(93)90014-F. 
7. Sohanker, A.; Norberg b, C.; Davidson, L.: Numerical simulation of unsteady low-Reynolds number flow around rectangular rods at incidence. J. Wind Eng. and Ind. Aerodyn. 69: 189-201 (1997). https://doi.org/10.1016/ S0167-6105(97)00154-2.

8. Mannini, C.; Soda, A.; Schewe, G.: Numerical investigation on the three-dimensional unsteady flow past a 5:1 rectangular rod. J. Wind Eng. and Ind. Aerodyn. 99(4): 469-482 (2011). https://doi.org/10.1016/j.jweia.2010.12.016.

9. Islam, S. U.; Zhou, C. Y.; Shah, A.; Xie, P.: Numerical simulation of flow past rectangular rods with different aspect ratios using the incompressible lattice Boltzmann method. J. Mech. Sci. Tech. 26(4): 1027 (2012). https://doi. org/10.1007/s12206-012-0328-4.

10. Huang, Z.; Xi, G.; Zhang, W.: Numerical simulation of spacing effects on the flow past two 2: 1 rectangular rods in tandem at $\mathrm{Re}=200$. In Proceedings of the ASME 2012 Fluid Eng. Summer Meeting (2012, July). https://doi. org/10.1115/FEDSM2012-72157.

11. Igarashi, T.: Characteristics of the flow around two circular rods arranged in tandem (1st report). Bulletin of the JSME, 27: 323-331 (1981). https://doi.org/10.1299/ jsme1958.24.323.

12. Zdravkovich, M. M.: The effects of interference between circular rods in cross flow. J. Fluids Struct. 1(2): 239-261 (1987). https://doi.org/10.1016/S0889-9746(87)90355-0.

13. Igarashi, T.; Suzuki, K.: Characteristics of the flow around three circular rods. Bulletin of JSME, 27(233): 2397-2404 (1984). https://doi.org/10.1299/jsme1958.27.2397.

14. Harichandan, A.; B.; Roy, A.: Numerical investigation of low-Reynolds number flow past two and three circular rods using unstructured grid CFR scheme. Int. J. Heat and Fluid Flow, 31(2): 154-171 (2010). https://doi.org/10.1016/j.ijheatfluidflow.2010.01.007

15. Ul-Islam, S.; Abbasi, W. S.; Rahman, H.; Naheed, R.: Numerical investigation of wake modes for flow past three tandem rods using the multi-relaxation-time lattice Boltzmann method for different gap spacing. J. Brazilian Soc. Mech. Sci. Eng. 38(3): 799-812 (2016). https://doi. org/10.1007/s40430-014-0282-4.
16. Wolf-Gladow, D.A.: Lattice-Gas Cellular Automata and Lattice Boltzmann Models-An introduction. Springer (2005)

17. Bhatnagar, P.; Gross, E. P.; Krook, M.: A model for collision processes in gases. I. Small amplitude processes in charged and neutral one-component system. Phys Review. 94: 511525 (1954). https://doi.org/10.1103/PhysRev.94.511.

18. Cheng, M.; Whyte, D. S.; Lou, J.: Numerical simulation of flow around a square rod in uniform-shear flow. J. FLUID STRUCT. 23: 207-226 (2007). https://doi.org/10.1016/j. jfluidstructs.2006.08.011.

19. Dazchi, Y.; Renwei, M.; Luo, L. S.; Wei, S.: Viscous flow computations with themethod of lattice Boltzmann equation. Prog. Aerospace Sci. 39: 329-367 (2003). https://doi. org/10.1016/S0376-0421(03)00003-4.

20. Robichaux, J.; Balachander, S.; Vanka, S. P.: Threedimensional Floquet instability of the wake of square rod. Phys. Fluids. 11(3): 560-578 (1999). https://doi. org/10.1063/1.869930.

21. Gera, B.; Sharma, P. K.; Singh, R. K.: CFD analysis of 2D unsteady flow around a square rod. Int. J. Appl. Eng. Res. 1(3): 602 (2010).

22. Abdollah, S.; Mahdi, A.;Noor Allah, R.: Experimental study of near wake flow behind a rectangular cylinder, American J. Applied Sci. 5: 917-926 (2008). https://doi.org/10.3844/ ajassp.2008.917.926.

23. Islam, S. Ul.; Zhou, C. Y.; Shah, A. and Xie, P.: Numerical simulation of flow past rectangular cylinders with different aspect ratios using the incompressible lattice Boltzmann method. J. Mech. Sci. Tech. 26:1027-1041 (2012). https://doi.org/10.1007/s12206-012-0328-4.

24. Igarashi, T.: Characteristics of the flow around two circular cylinders arranged in tandem ( $1^{\text {st }}$ report). Bulletin of the JSME, 24: 323-331 (1981). https://doi.org/10.1299/ jsme1958.24.323.

25. Kim, M. K.;Kim, D. K.; Yoon, S. H. Lee, D. H.: Measurements of the flow fields around two square cylinders in a tandem arrangement. J. Mech. Sci.Tech. 22: 397-407 (2008). https://doi.org/10.1007/s12206-007-1041-6. 